

\section{SOBRE OS AUTORES}

João Victor Gomes dos Santos | contato@votidesign.com

Lattes: http://lattes.cnpq.br/0165600236611511

Designer de Produtos formado pela Unesp - Campus Bauru. Estagiou no setor de desenvolvimento de produtos e gerenciamento de produção na Paus + Grün Woodwork Design Inc. em Toronto - Canadá e atualmente é Mestrando em Planejamento de Produto do Programa de Pós Graduação em Design da Unesp - Bauru (Capes 6). É integrante do grupo de pesquisa CNPq Tecnologia e Manufatura de Materiais Avançados onde desenvolve pesquisas na área de desenvolvimento de novos produtos com aplicação de biocompósitos e tecnologias de prototipagem virtual e física, modelagem virtual 3D e gestão de produção através de softwares CAD, CAE e CAM.

Marco Antonio dos Reis Pereira | pereira@feb.Unesp.br Lattes: http://lattes.cnpq.br/3710990353238773

Graduação em Engenharia Agrícola pela Universidade Estadual de Campinas (1982), mestrado em Agronomia (Irrigação e Drenagem) pela Universidade Estadual Paulista Júlio de Mesquita Filho (1992), doutorado em Agronomia (Irrigação e Drenagem) pela Universidade Estadual Paulista Júlio de Mesquita Filho (1997), especialização em Irrigação e Drenagem pela Universidade Federal de Viçosa (1989) e especialização em bambu no "2001' Tcdc International Training Course On Bamboo" pelo CBRC - China National Bamboo Research Center (Hangzhou-China). Atualmente é professor adjunto e livre docente em Design e Construção com Bambu na Universidade Estadual Paulista Júlio de Mesquita Filho (Unesp campus de Bauru-SP). Docente do curso de Engenharia Mecânica, ministra aulas nas disciplinas Mecânica dos Fluídos, Laboratório de Fenômenos de Transporte, Laboratório de Mecânica dos Fluídos e Engenharia de Irrigação desde o ano de 1988 no curso de Engenharia Mecânica. Docente na Pós-Graduação do curso de Design da FAAC/Bauru onde orienta e ministra a disciplina Design e Construção com Bambu.

Fausto Orsi Medola | http://lattes.cnpq.br/6962871803093697 | fausto.medola@faac.unesp.br

Professor Assistente Doutor - FAAC/UNESP - Departamento de Design - Campus Bauru, e vice-coordenador do Programa de Pós-graduação em Design - FAAC/UNESP. Doutor em Ciências (ărea de concentração: Bioengenharia) pelo Programa de Pós-graduação Interunidades Bioengenharia - EESC/FMRP/IQSC - USP (2013), com estágio de doutorado sanduíche na Georgia Institute of Technology, Atlanta, EUA. Mestrado em Bioengenharia pelo Programa de Pósgraduação Interunidades em Bioengenharia - EESC/FMRP/IQSC - USP, e graduação em Fisioterapia pela Universidade Estadual de Londrina (2003). É líder do Grupo de Pesquisa Design e Tecnologia Assistiva, e membro pesquisador no Grupo de Pesquisa Desenho Industrial: Projeto e Interfaces. Realiza pesquisas na área de Desenho Industrial, Design de Produto e Ergonomia, atuando principalmente nos temas: pesquisa e desenvolvimento de equipamentos de tecnologia assistiva, design ergonômico, biomecânica, cadeira de rodas, acessibilidade e design inclusivo.

Luis Carlos Paschoarelli | Lattes: http://attes.cnpq.br/8521603444193259 | paschoarelli@faac.unesp.br

Bolsista de Produtividade em Pesquisa do CNPq - Nível 1D (CA Desenho Industrial. É Professor Titular no Departamento de Design da UNESP (2017); Livre-Docente em Design Ergonômico pela UNESP (2009); possui Pós-doutorado em Ergonomia (2008) pela ULISBOA; Doutorado em Engenharia de Produção (2003) pela UFSCar; Mestrado em Projeto, Arte e Sociedade - Desenho Industrial (1997) e graduação em Desenho Industrial (1994) pela UNESP. É co-lider no Grupo de Pesquisa Design Ergonômico: Projeto e Interfaces onde coordena os projetos de pesquisa: Design Ergonômico: avaliação e intervenção ergonômica no projeto, Design Ergonômico: metodologias para a avaliação de instrumentos manuais na interface homem X tecnologia e Contribuições do Design Ergonômico na pesquisa e projeto de equipamentos para a reabilitação de pessoas com capacidades específicas. Está lotado no Laboratório de Ergonomia e Interfaces - Departamento de Design, onde atua como docente no curso de graduação em Design e do Programa de Pós-graduação em Design da UNESP. Tem experiência na área do design, ergonomia, design ergonômico, design de produto, design gráfico e tecnologia assistiva. Atualmente é "Ergonomista Sênior" da ABERGO - Associação Brasileira de Ergonomia. 


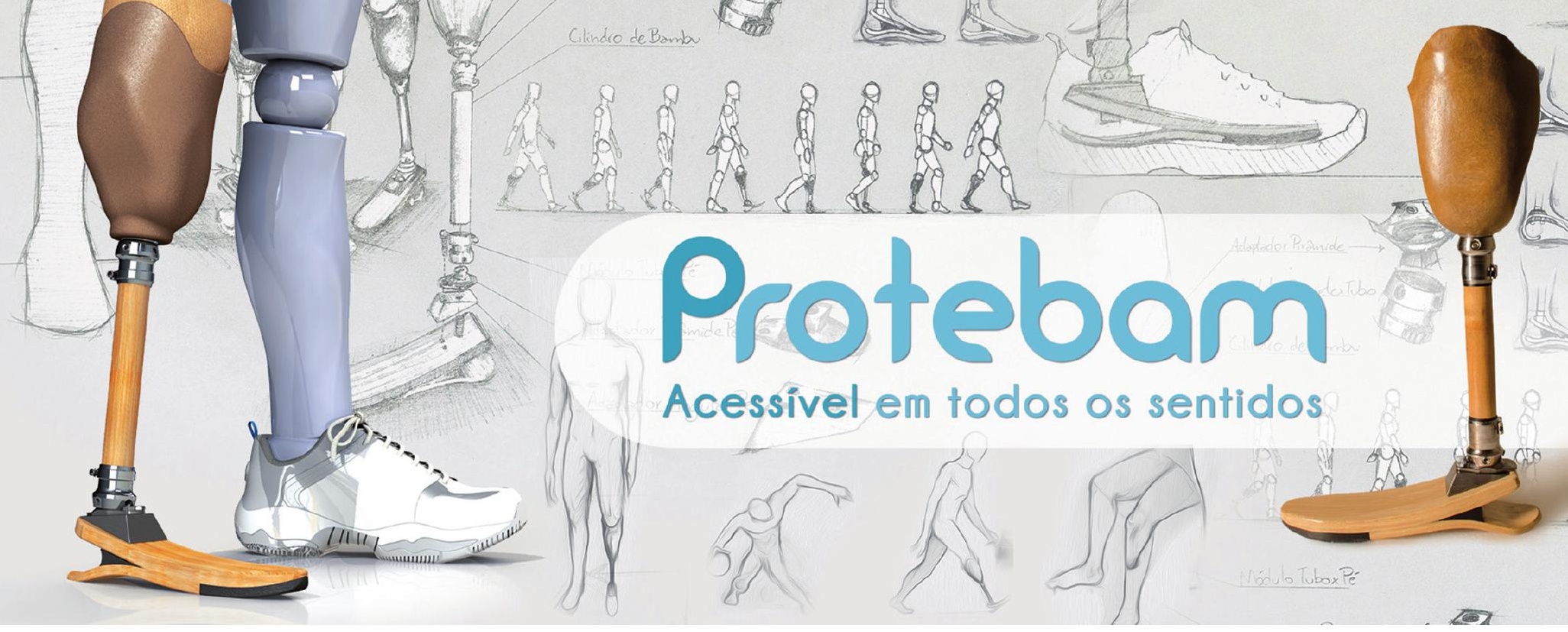

\title{
Design sustentável aplicado ao projeto de produtos assistivos (proteses) fabrícados com biocompósitos
}

\author{
Sustainable design applied to the project of a \\ prosthesis manufactured with biocomposites
}

João Victor Gomes dos Santos, Marco Antonio dos Reis Pereira, Fausto Orsi Medola, Luis Carlos Paschoarelli

\section{Resumo}

O crescimento de estudos que buscam solucionar problemas vivenciados por pessoas com deficiências é proporcional ao aumento mundial desta faixa da população. Além dos estudos desta área, crescem também os estudos que buscam a preservação do meio ambiente, como pesquisas de novos materiais biodegradáveis que utilizam matéria prima de fontes renováveis. Buscando atender essas demandas foi desenvolvido um novo biocompósito composto por materiais de fonte renovável que, além do baixo custo e impacto ambiental mínimo, apresentou trabalhabilidade adequada para fabricação de produtos com formas orgânicas e personalizadas, como órteses e soquetes para próteses. 0 material é composto por fibras de bambu e resina poliuretana à base de mamona que, devido às suas propriedades térmicas, também contribui para a redução do desconforto térmico frequentemente mencionado por usuários de próteses.

Palavras-chaves: Design sustentável; Prótese transtibial; Biocompósito; Bambu.

\begin{abstract}
The growth of studies that seek to solve problems experienced by people with disabilities is proportional to the worldwide increase of this population. In addition to studies in this area, there are also studies that seek to preserve the environment, such as research on new biodegradable materials that use raw materials from renewable sources. In order to meet these demands, a new biocomposite composed of renewable source materials was developed, which, in addition to the low cost and minimal environmental impact, presented adequate workability for manufacturing products with organic and customized shapes, such as orthoses and sockets for prostheses. The material is composed of bamboo fibers and polyurethane resin based on castor oil, which, due to its thermal properties, could also contribute to the reduction of the thermal discomfort of the wearers of protheses.
\end{abstract}




\section{INTRODUÇÃO}

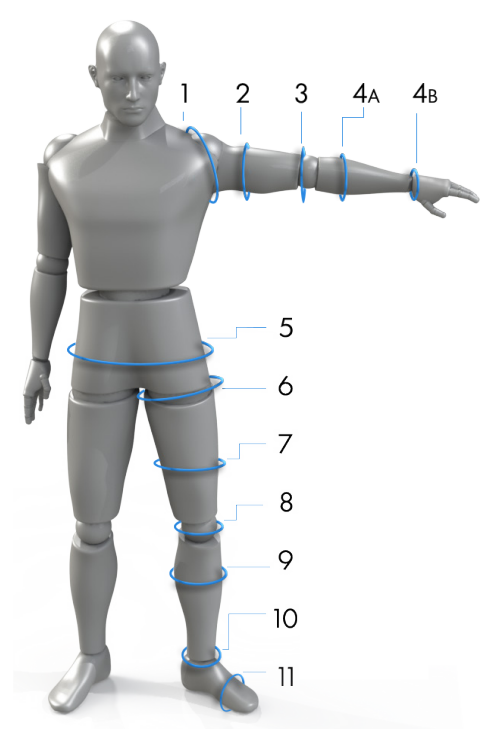

Figura 1: Niveis de Amputação. O nivel 9 corresponde à Amputação Transtibial.

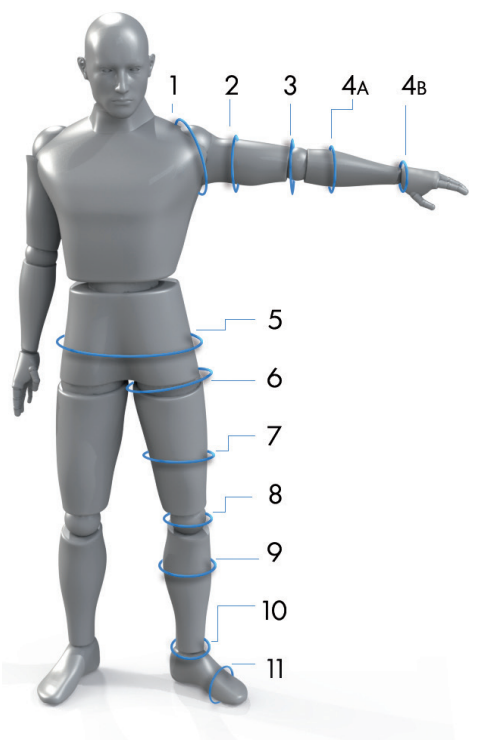

Figura 2: Prótese Transtibial Endoesquelética com destaque para o componente "Soquete".
A quantidade de estudos envolvendo pessoas com deficiência, bem como outras minorias populacionais, vem crescendo em todo o mundo, o que contribui para aumento do razoável acervo de conhecimento existente sobre a área (IIDA, 2016). Estes estudos contribuem para o desenvolvimento de novas técnicas e produtos que, com o auxílio das áreas ortopédicas e participação efetiva do fisioterapeuta, resultam na criação de novos componentes pré-fabricados e padronizados, que possibilitam uma melhor reabilitação funcional dos usuários e reduzem suas limitações físicas (BOCCOLINI, 2000).

Dentre as limitações físicas, a amputação pode ser considerada uma das mais difíceis em termos de reabilitação, uma vez que é permanente e ainda exige procedimentos invasivos de longo tempo de reabilitação. As causas mais comuns das amputações são as doenças vasculares periféricas, habitualmente decorrentes de patologias como a diabetes, ou hábitos como o tabagismo, os quais representam cerca de $80 \%$ das causas. Outras condições, tais como acidentes automobilísticos e outros, representam $20 \%$. Dentre todas as amputações, as de membros inferiores predominam em 85\% dos casos (TOOMS, 1987) sendo a amputação a nível transtibial (Adicionar após esta palavra: (correspondente ao Nível 9 da Figura 1) a mais frequente em amputados de causas traumáticas (TINTLE, et al. 2010). No Brasil, a incidência de amputações é 13,9 por 100.000 habitantes/ano (CARVALHO, 2003), sendo a de nível transtibial, realizada entre a articulação do tornozelo e a do joelho, a segunda maior incidência em membros inferiores (SANTOS et al. 2010). Segundo estudos de 2005, nos Estados Unidos, existem cerca de 1,6 milhões de pessoas amputadas e a estimativa é que este número aumente para 3,6 milhões até 2050 (ZIEGLERGRAHAM, et al. 2008).

Nas próteses para membros inferiores, o soquete (Figura 2) é a parte mais importante, já que realiza a interface entre a prótese e o coto, ou clinicamente denominado "coto". Sua eficiência envolve não somente a distribuição da força e a pressão no coto, mas também a satisfação do usuário quanto ao conforto térmico. Segundo a revisão literária feita por Ghoseiri e Safari (2014) dentre 38 estudos revisados, 27 identificaram a prevalência de calor e/ou desconforto dentro do soquete. Das pessoas com amputação estudadas, $53,68 \%$ queixam-se de desconforto térmico no interior de suas próteses, independentemente do seu tipo ou nível de amputação. 
Além da influência na percepção do usuário, o setor de protetização também causa impactos no meio ambiente visto que os materiais mais comuns para fabricação de próteses e órteses atualmente são compósitos sintéticos (Figura 3) constituídos, em grande parte, por fibra de carbono, fibra de vidro, resina epóxi e outros materiais não sustentáveis (MANOHAR, 2016). Somado a isso, por serem fabricados especificamente para cada indivíduo, os soquetes não podem ser reutilizados, sendo, portanto, descartados após sua vida útil ou quando não servem mais no membro do usuário, como no caso de crianças em fase de crescimento e usuários que ganham ou perdem peso.

Portanto, considerando a relevância do problema, sua prevalência em uma parte considerável da população mundial e ainda a previsão de seu aumento contínuo (RESNIK e BORGIA, 2011), é evidente a existência de uma demanda por estudos que contribuam para o desenvolvimento de soluções nesta área do design. Nessa perspectiva, através da aplicação de metodologias baseadas no Design Sustentável, este estudo propõe parâmetros para o desenvolvimento de produtos assistivos, cuja produção e materiais sejam sustentáveis e suas propriedades mecânicas e térmicas proporcionem segurança e conforto aos usuários, atingindo, assim, o equilíbrio entre os três principais requisitos de um produto sustentável: viabilidade econômica, consciência ambiental e a responsabilidade social (LÖBACH, 2001).

\section{PRÓTESES E ÓRTESES}

Próteses e órteses são artefatos destinados a suprir as necessidades de quem possui um membro, ou parte deste, gravemente acometido ou com alguma deficiência. Enquanto as órteses corrigem uma alteração morfológica do órgão, a prótese substitui o órgão ou parte deste em casos de amputação (Figura 4).

Dentre as amputações de membros inferiores, a amputação transtibial é considerada uma das que possuem o melhor prognóstico para uso de prótese e nível posicional para reabilitação (O'SULLIVAN \& SCHIMITZ, 1993). Isso se deve ao fato de que além do comprimento do coto facilitar o encaixe da prótese, a amputação ocorre abaixo do joelho, não havendo, portanto, a necessidade de equipamentos que simulem esta articulação. Estes fatores resultam em uma perturbação menor na marcha do indivíduo e um gasto de energia para deambulação inferior, quando comparados com niveis mais proximais de amputação (DOUGHERTY, 2001; PURRY \& HANNON, 1989).

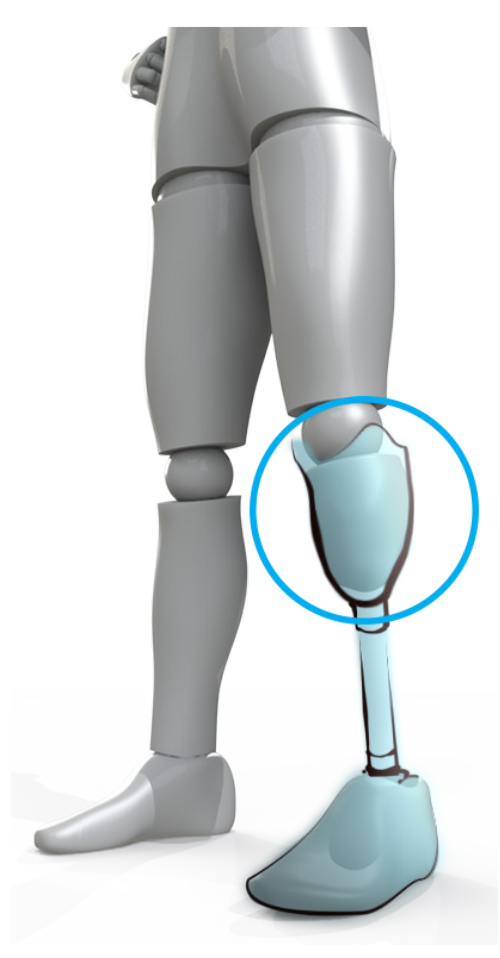

Figura 3: Tipos de próteses e materiais sintéticos comumente utilizados.

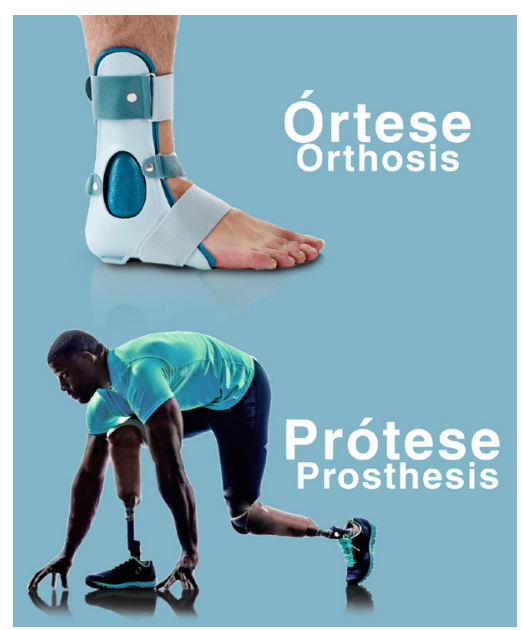

Figura 5: Órtese = Correção de um membro $\mid$ Prótese $=$ Substituição de um membro ou parte deste. 
Apesar de essencial, o uso contínuo de uma prótese ou órtese pode causar desconforto e provocar problemas de pele, principalmente para amputados de membros inferiores cujo peso do corpo é sustentado, em grande parte, pelo coto (SCHOPPEN et al. 2003). Mesmo com o constante esforço de pesquisadores e profissionais de reabilitação nessa área, ainda não foram criadas soluções que substituam os membros perdidos de maneira eficiente e pouco se sabe sobre as temperaturas da pele do coto dentro do soquete ou a diferença entre eles (KLUTE et al. 2007).

Diversos estudos que envolvem a reabilitação de amputados mostram que os usuários de próteses reclamam do desconforto ocasionado pelo uso dos equipamentos (Tabela 1) e consideram este aspecto um dos principais a ser considerado no uso de próteses (PINTO et al. 1998; MAK, 2001). Outros estudos demonstram que problemas de pele no coto em amputados de membros inferiores são observados com frequência na prática clínica (LYON, et al. 2000; KOC, et al. 2008). Lake (1997) observou em seu estudo que de $60 \%$ a $70 \%$ das pessoas com amputação relataram alta transpiração dentro do encaixe da prótese como um grave problema.

Dentre outros fatores, esse desconforto é ocasionado pela baixa permeabilidade dos materiais utilizados pela maioria dos soquetes atuais que funcionam como uma barreira física que dificulta a transferência do calor e de evaporação de dentro do soquete (HACHISUKA et al. 2001). Somado a isso, a falta de cuidados com a pele do coto pode acarretar em irritações e interferir no uso diário da prótese tornando-se um grande incômodo (LEVY, 1995). Essas complicações podem ocorrer de forma mecânica (calos, hiperplasia verrucosa e outros), por reações alérgicas (edema, eczema, dermatite de contato e outros) (Figura 5) e/ou por infecções bacterianas e fúngicas (DUDEK, et al. 2005; MEULENBELT, et al. 2011) e ocorrem devido ao contato justo entre o coto e o soquete. Ambas interfaces - coto e soquete - são responsáveis pelo controle da prótese enquanto o usuário está em pé, estático ou em movimento. $O$ soquete deve realizar a transferência adequada das forças na sua interface com o coto, dissipando a força por todo o coto, evitando, assim, o acúmulo de forças em um determinado ponto (KLUTE et al. 2009).

$\mathrm{O}$ ajuste e a suspensão adequados do soquete requerem um contato total da superfície do componente com o coto, limitando, dessa maneira, a circulação de ar na interface da pele com o soquete. 0 calor no seu 
interior, dentro do qual a pele tende a transpirar mais do que o habitual (LEVY, 1995), deixa a pele levemente úmida o que a torna mais suscetível a bolhas do que a pele molhada ou seca (NAYLOR, 1955). Além dos fatores já citados, a baixa permeabilidade dos materiais comumente utilizados na fabricação dos componentes (plástico, silicone e outros materiais inorgânicos) também contribui para a retenção da umidade na interface soquete $x$ coto. Segundo Klute et al. (2007) a transferência efetiva deste calor retido no soquete poderia reduzir a temperatura da pele envolvendo o processo controlado pela condutividade térmica, como forro, meias e camadas de soquete. Assim, é possível concluir que, ao diminuir a temperatura e a umidade da pele no interior do soquete, pode-se reduzir o desconforto e a incidência de lesões (PEERY et al. 2006); e esse objetivo pode ser alcançado através da aplicação de materiais cuja transferência de calor seja efetiva e reduza a temperatura da pele, envolvendo o processo controlado pela condutividade térmica, como forro, meias e camadas de soquete (KLUTE et al. 2007).

\section{DESIGN SUSTENTÁVEL}

Nas últimas décadas, projetos de diversas áreas do conhecimento passaram a adotar critérios ecológicos cada vez mais rigorosos em seu desenvolvimento, abrangendo projetos gráficos, territoriais, arquitetônicos e de bens de consumo, em busca da melhoria na qualidade de vida das populações e na disponibilidade futura de matéria-prima e energia. Estas atividades projetuais, que adotam conceitos ecológicos em seu desenvolvimento, podem ser representadas pelo termo ecodesign (Figura 6) que, segundo Fiksel (1996), pode ser considerado como uma estratégia de projeto, cujo desempenho respeita aspectos ambientais, de saúde e segurança, ao longo de todo o ciclo de vida de um produto, tornando-os ecoeficientes.

O Programa das Nações Unidas para o Meio Ambiente (PNUMA) propõe oito níveis de implantação do ecodesign que abrangem desde a preferência pela utilização de biomateriais de baixo impacto na produção, ou seja, materiais renováveis e degradáveis (PELTIER; SAPORTA, 2009), até o planejamento do descarte, reciclagem ou reutilização do produto. Todos os aspectos devem levar em conta a otimização da aplicação da matériaprima e menor geração de resíduos, bem como a reconsideração das funções do produto, podendo ser eliminadas as que não agregam valor ao

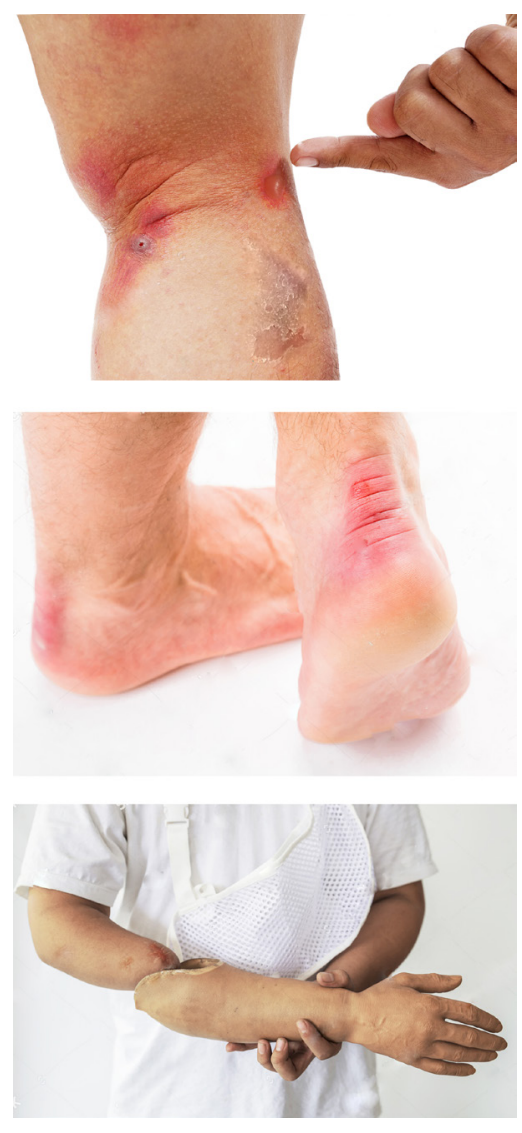

Figura 5: Problemas de Pele causados pelo uso de próteses elou órteses.

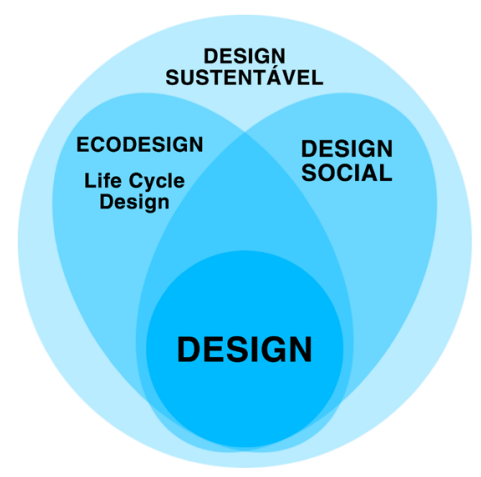

Figura 6: Problemas de Pele causados pelo uso de próteses e/ou órteses. 
produto, que tenham apenas funções estéticas, e utilizem matéria-prima acima do necessário sem perder com isso as características originais.

Esta condição pode ser representada pelo Life Cycle Design, que recomenda a constante avaliação de um projeto em relação às suas implicações ambientais, seja um produto ou serviço, por todo o seu ciclo de vida; e que pode ser determinado pela sua pré-produção, produção, distribuição, uso e descarte. A extração dos recursos necessários para produção dos materiais ("nascimento") também é considerada neste método. Bem como o tratamento final dos materiais após o uso ("morte"), marcando o início e o fim da vida de um produto cujo processo de produção é constituído por uma série de atividades e processos, que absorvem matéria e energia, transformando-se, gerando resíduos e emitindo substâncias diversas. Em um sistema de produção ecologicamente correto, essas emissões são controladas e os resíduos reintegrados à linha de produção, fechando o ciclo.

Todos esses aspectos podem ser resumidos por um único termo, o Design para Sustentabilidade. Segundo Cavalcanti (2012), além de considerar o impacto ambiental que um produto ou sistema pode causar o Design para Sustentabilidade também incorpora aspectos econômicos como Geração de Empregos, Desenvolvimento de Recursos Humanos, Potencialização do Mercado Interno, e outros fatores relacionados ao mercado.

Além desses enfoques, o Design para a Sustentabilidade também tem comprometimento com a Sociedade, buscando sempre combater a Desigualdade Social, a Pobreza e Marginalização das Minorias. No âmbito dos produtos assistivos, o impacto social ocorre não somente nas pessoas com deficiência, mas em outras populações marginalizadas, como a população de baixa renda. Conforme a Organização Mundial de Saúde (OMS) existe uma forte relação entre pobreza e deficiência, sendo que $80 \%$ das pessoas com deficiência no mundo vivem com baixa renda (ELIAS; MONTEIRO e CHAVES, 2007). Isso mostra que uma amputação para indivíduos de baixa renda e escolaridade se torna algo preocupante, visto que são pessoas que necessitam basicamente ter seu estado físico saudáveis para o desempenho de seu trabalho e, consequentemente, de seu sustento (BOCCOLINI, 2000) e assim contribuir, também, com a economia local. Dessa maneira, um produto verdadeiramente sustentável, segundo Löbach (2001), é aquele que respeita todos os requisitos do Tripé da Sustentabilidade (Figura 7), ou seja, viabilidade econômica, consciência ambiental e a responsabilidade social. 


\section{BIOCOMPÓSITOS}

O termo compósito refere-se a um material formado pela mistura física de dois ou mais materiais, imiscíveis entre si, combinados para formar um novo material que possui propriedades diferentes em relação aos seus componentes puros (ASTM, 2007). Já os biocompósitos são constituídos principalmente pela combinação de reforços biodegradáveis de origem natural e renovável como, por exemplo, fibras lignocelulósicas e semelhantes, e a matriz, que pode ser um polímero biodegradável como óleos vegetais ou amidos (KHAN et al. 2017).

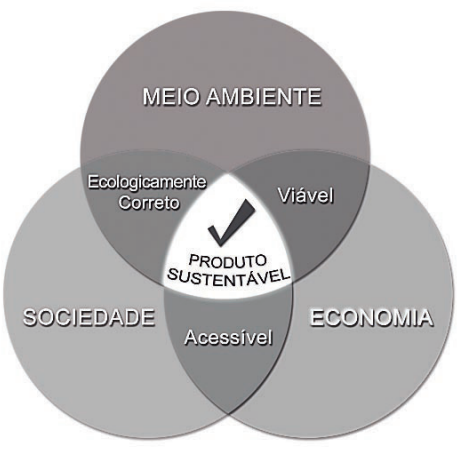

Figura 7: Tripé da sustentabilidade. Fonte: Adaptado de Kraemer, 2003.

Atualmente, ao se comparar os biopolímeros aos termoplásticos convencionais geralmente seu custo é elevado e suas propriedades inferiores o que tornam sua aplicação menos vantajosa (PELLICANO et al. 2009). Portanto, a criação de biocompósitos com inserção de fibras vegetais em matrizes poliméricas, além de benéfico ao meio ambiente, pode ser uma alternativa para melhorar as propriedades do material e ao mesmo tempo reduzir o custo tornando-os competitivos em relação aos materiais convencionais.

Além de ecologicamente corretos, recicláveis, potencialmente abundantes e econômicos em comparação às fibras sintéticas, os compósitos poliméricos reforçados por fibras são adequados a algumas aplicações específicas pela possibilidade de serem personalizados de acordo com a necessidade de aplicação e por possuírem formato complexo não obtido facilmente por outros materiais. Além disso, sua matriz polimérica the confere elevada resistência química, elevado módulo elástico e resistência à tração específica (AGARWAL \& BROUTMAN, 1990), resultando em materiais resistentes, econômicos e leves, ideais para uso como reforço em compósitos para ortopedia e protetização (KRAMER et al. 2015).

Na fabricação de compósitos reforçados por fibras de bambu, as lâminas e fibras são retiradas da parte do bambu que contém maior quantidade de fibras (região externa próxima à casca) e unidas por adesivos como, por exemplo, o epóxi. Os adesivos mais utilizados para a fabricação desses compósitos laminados de bambu são geralmente os mesmos utilizados para painéis de madeira. Estes podem ser classificados segundo a sua composição química ou conforme o regime de trabalho ao qual o produto será submetido 


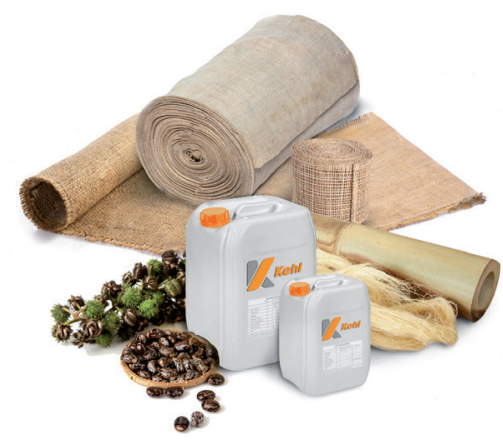

Figura 8: Matéria prima da matriz do biocompósito (sementes de Ricinus communis) e exemplos de fibras naturais como linho, cânhamo, kenaf, algodão e bambu.
(LIMA, 2006). Os adesivos mais utilizados são: melamina, ureia-formaldeído, fenol-formaldeído, resorcinól-formaldeído e epóxi (LÓPEZ, 2003).

Como exemplo de protetização (descrito abaixo), foram utilizados dois tipos de biocompósitos: o BLaC, Bambu Laminado Colado e o BRFB, Biocompósito Reforçado por Fibras de Bambu. 0 primeiro refere-se a ripas obtidas da área próxima à casca do bambu, as quais são coladas e prensadas face-a-face com adesivo. Já no BRFB, são utilizadas fibras e pó de bambu resultantes do trituramento dos resíduos gerados pela produção do BLaC. Devido à natureza sustentável da protetização em questão, os adesivos previamente citados não foram considerados adequados para aplicação neste projeto devido à sua composição química. Dessa maneira, recorreu-se ao adesivo do tipo poliuretano à base de óleo de mamona (Figura 8) por se tratar de um produto cuja matéria-prima é de fonte renovável e apresentar propriedades físicas, químicas e mecânicas adequadas à pesquisa.

A mamona (Ricinus communis L.) é uma planta da família das Euforbiáceas e seu principal produto é o óleo extraído da sua semente conhecido como óleo de rícino que, devido a sua composição química peculiar, é muito utilizado na fabricação de biodiesel e também na indústria química. 0 poliuretano (PU) à base de óleo de mamona pode ser classificado como uma resina termofixa líquida de baixa a alta viscosidade. Trata-se de um polímero bicomponente composto por um pré-polímero e um poliol extraído do óleo da semente da mamona. Por ser proveniente de fonte renovável, constitui uma excelente alternativa para o desenvolvimento de biocompósitos, além disso, segundo Godoy (2007) é completamente atóxica por não apresentar materiais voláteis e metais pesados em sua composição; e não precisar de solventes para aplicação.

Segundo a American Society of Materials (ASM, 2001), dentre os polímeros comerciais utilizados como matriz para a obtenção de compósitos poliméricos reforçados com fibras, as resinas epóxi e poliuretano destacam-se devido à grande variedade de escolha dos reagentes, o que permite obter materiais com diferentes propriedades físicas e químicas.

Segundo Targa (2011), a interação química dos grupos isocianatos da resina com grupos funcionais presentes nos materiais lignocelulósicos do bambu podem facilitar a adesão interfacial entre estas matrizes e as 
fibras. Essa melhor adesão permite uma melhor transferência da carga para fibras ao mesmo tempo em que oferece proteção contra abrasão e ataque de agentes externos (MAHAJAN e AHER, 2012).

\section{PROTETIZAÇÃO: PROCEDIMENTOS METODOLÓGICOS}

Para exemplificar os aspectos teóricos descritos anteriormente, foi desenvolvido um protótipo de prótese transtibial. Na etapa de desenvolvimento do produto, foram analisados diversos tipos de próteses e órteses para aplicação do biocompósito. Após essa análise, foi selecionada a prótese transtibial do tipo endoesquelética, por se tratar do tipo mais comum entre amputações de membros inferiores e considerada uma das que possuem o melhor prognóstico para uso de prótese e nível posicional para reabilitação. Trata-se de uma prótese modular composta basicamente por três componentes: Soquete, Tubo e Pé Protético e a conexão entre os componentes é realizada por meio dos componentes modulares.

As peças foram então projetadas a partir das medidas dos componentes modulares para garantir as tolerâncias corretas na montagem. Quanto ao soquete, por ser um componente personalizado de acordo com o coto do usuário, foi utilizado um manequim em polipropileno para simular o usuário que, além de possibilitar a simulação da produção do molde para o soquete (Figura 9), foi utilizado para demonstração de uso do protótipo. O manequim foi adaptado seguindo as recomendações referentes à amputação transtibial de maneira a simular um usuário amputado. Para tanto, foi realizado um corte à aproximadamente $12 \mathrm{~cm}$ abaixo da patela e a abertura foi preenchia com um bloco de espuma de PU rígida e massa plástica para acabamento.

Figura 9: Manequim com adaptação e confecção do molde negativo do membro residual.
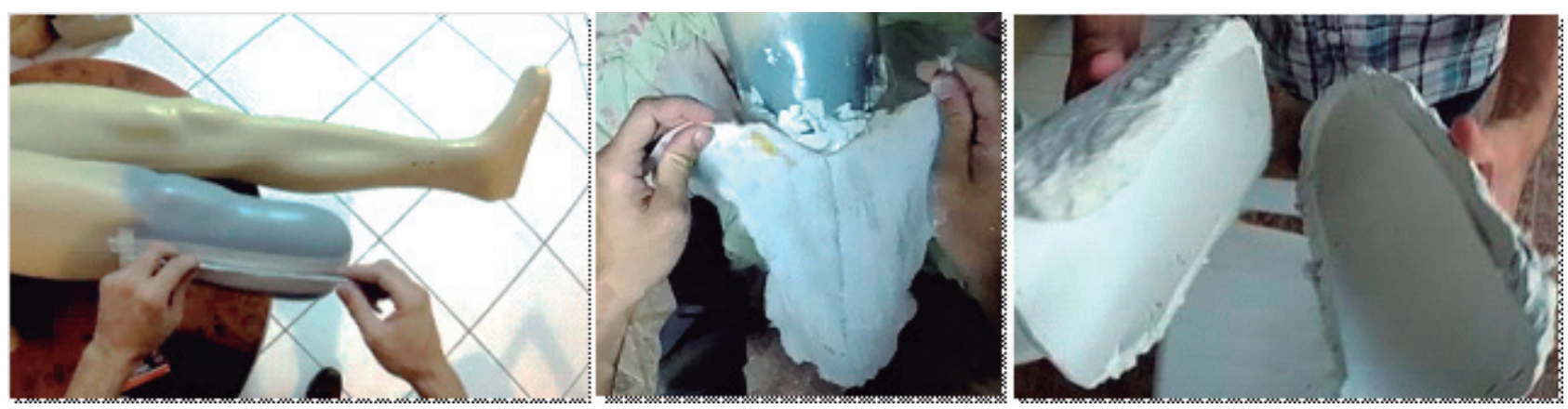


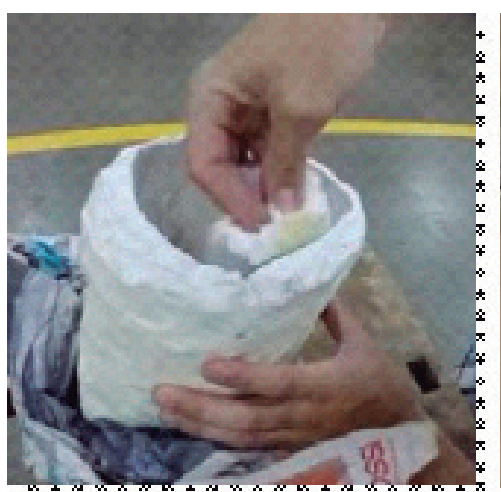

Figura 10: Preparação do molde

negativo e do silicone para confecção do molde positivo.
Figura 11: Colheita, Destopo e Refilação do colmo em Serra Circular.

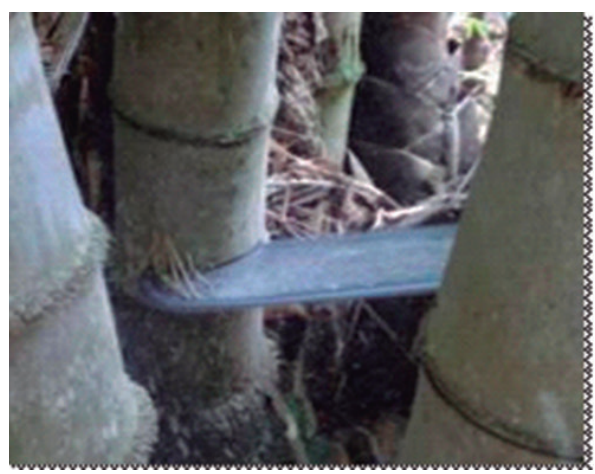

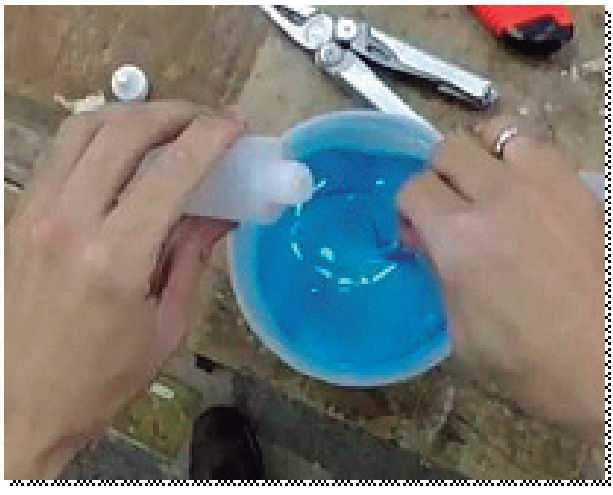

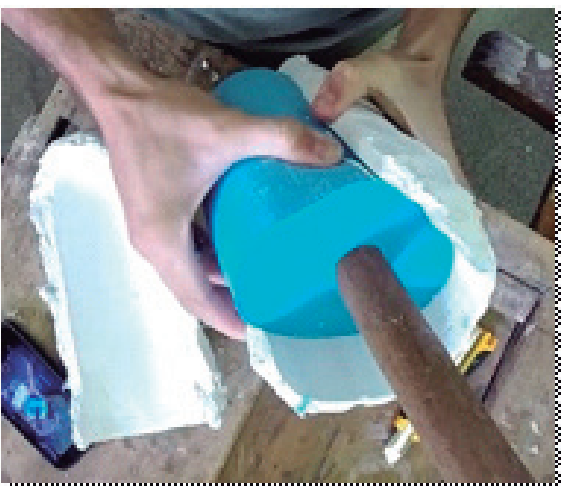

Depois da secagem das duas partes do molde negativo de gesso, ambas foram unidas e preparadas para adição do silicone líquido com catalisador para obtenção do molde positivo para laminação do soquete. Um cilindro de madeira foi inserido no silicone ainda líquido para facilitar a retirada do molde (Figura 10).

O bambu utilizado neste projeto pertence à espécie Dendrocalamus asper, de 3,5 anos de idade. Conhecido como bambu gigante ou ásper, é uma espécie de bambu entouceirante, de grande porte cujos colmos podem variar de 20 a 30 metros, com diâmetros entre 8 a 20 cm, espessura da parede de 1 e 2 cm, e os internos com distâncias de 20 a 45 cm.

Após a sua colheita, tratamento e secagem, que ocorreu no Plantio Experimental da Unesp - Bauru, o colmo foi encaminhado para a Serra Circular onde foi realizado o destopo de acordo com as medidas estipuladas pelos projetos da prótese (Figura 11). Os resíduos dos processos principais de obtenção das ripas para fabricação do BLaC foram reservados para obtenção das fibras que foram utilizadas para fabricação do soquete.
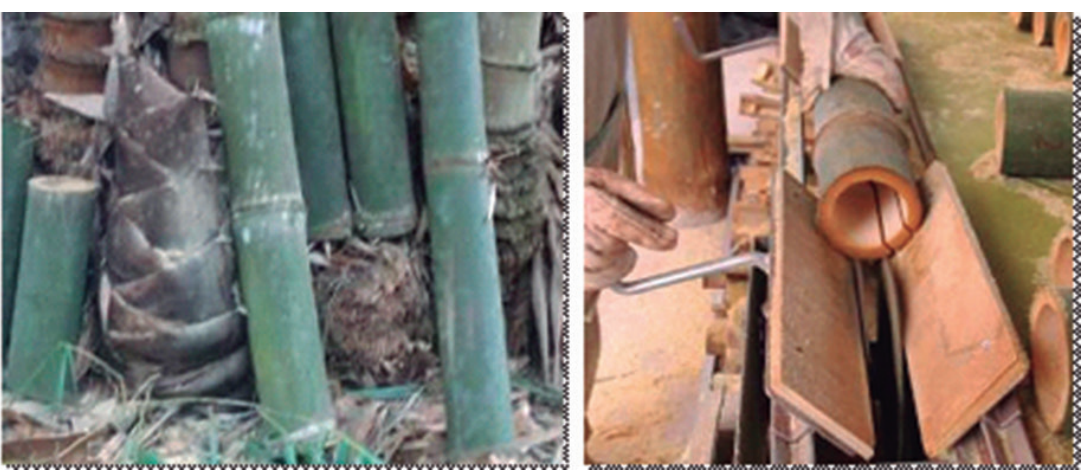

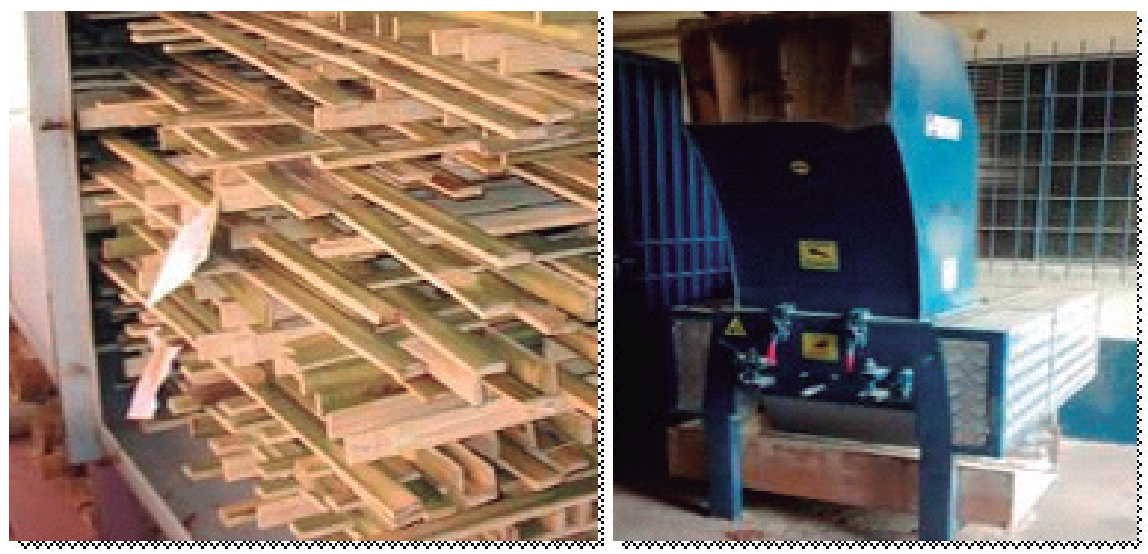

Os resíduos gerados pelo processo de obtenção das ripas do $\mathrm{BLaC}$

(Figura 12) foram coletados e triturados em um moinho de martelos. As fibras foram então peneiradas em uma Peneira Vibratória Mecânica. Após a separação, as fibras foram secas em uma Estufa Industrial com circulação e renovação de ar forçado por um período de 12 horas a $70 \pm 2{ }^{\circ} \mathrm{C}$ revirando cada 4 horas.

Posteriormente ao preparo da matéria-prima, foi iniciada a fabricação dos biocompósitos. A produção do BLaC e do BRFB ocorreu paralelamente para potencializar o tempo de produção, no entanto, este trabalho apresenta somente o processo de produção do BRFB visto que o foco é a produção do soquete. 0 poliol e o isocianato, componentes da resina de mamona, foram pesados na proporção 1:2, misturados manualmente com as fibras e a mistura foi laminada manualmente no molde positivo de silicone da prótese transtibial (Figura 13).
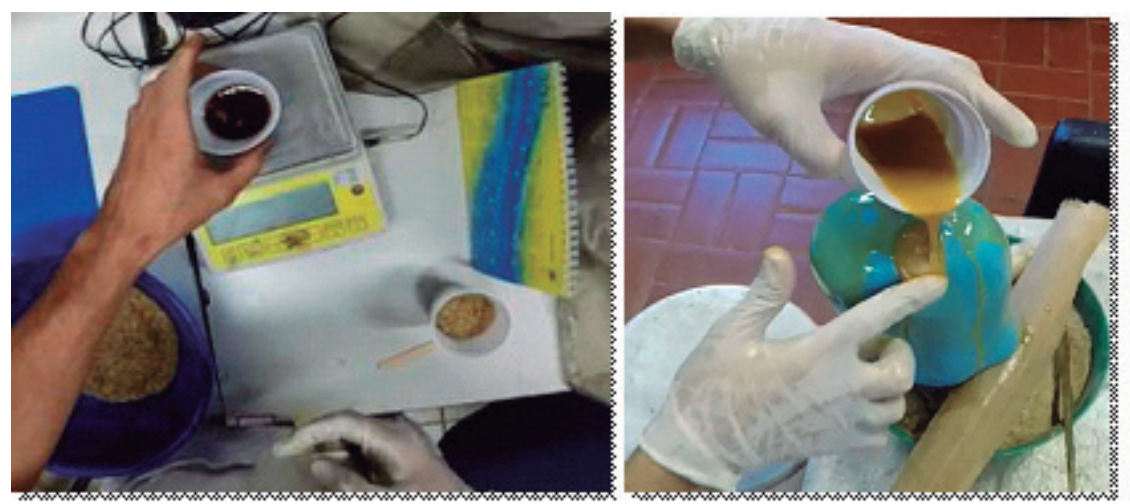

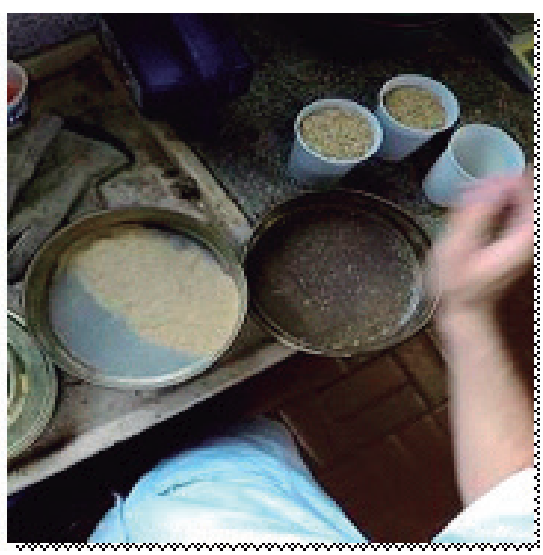

Figura 12: Ripas obtidas, moinho de martelos e fibras obtidas pela peneira.
Figura 13: Pesagem dos componentes da resina, preparação do molde e laminação.

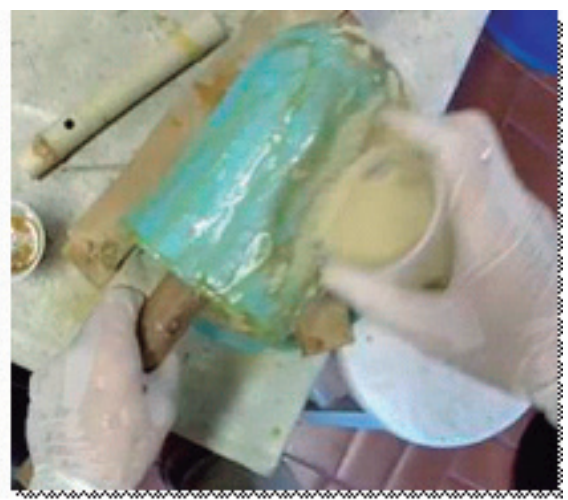




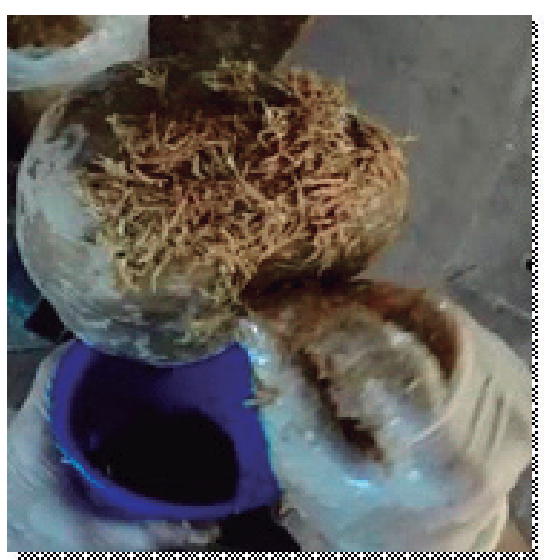

Figura 14: Laminação da camada estrutural e instalação do componente modular.
Figura 15: Retirada do soquete, acabamento e montagem do protótipo.

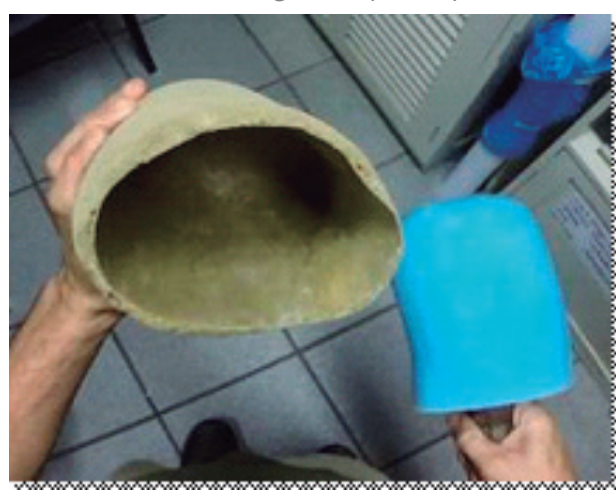

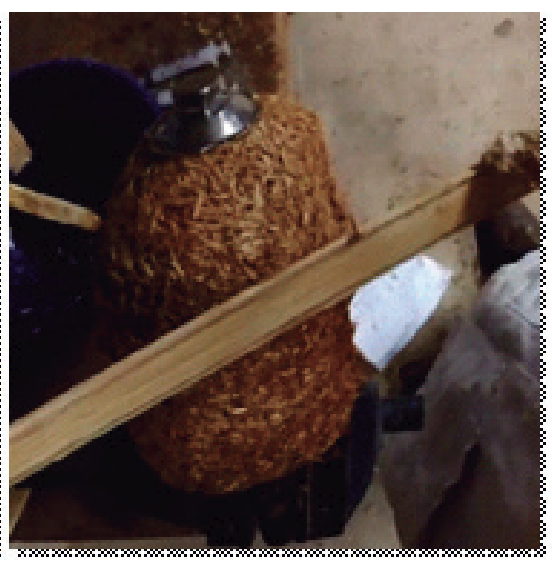

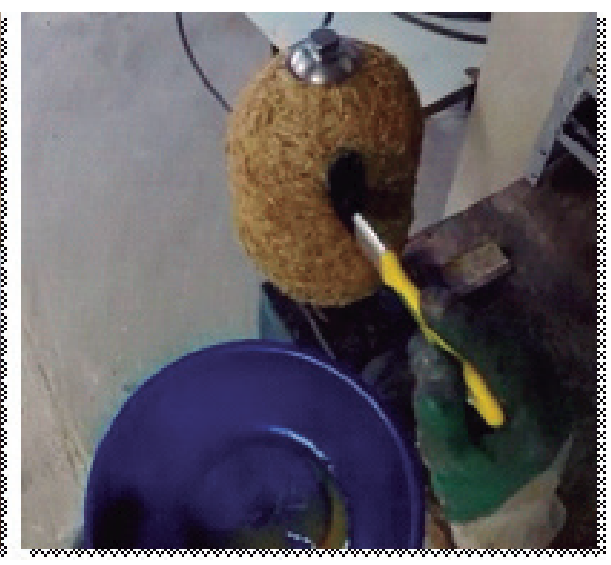

Logo após a laminação, o molde foi prensado com auxílio de um rolo para melhor adesão das fibras e remoção de bolhas. Além desta camada, o molde recebeu mais duas camadas de resina e fibra respeitando o tempo de cura de 24 horas entre camadas. Para a fabricação do cartucho foram utilizados três tipos de fibras. Para o preparo da camada de estrutura foram utilizadas fibras mesh 6 misturadas com mesh 8 na proporção $1 \times 1$. Para a mistura da camada de acabamento foi utilizado o pó resultante do peneiramento. Para ambas as misturas, foram utilizadas a proporção de resina 1:2 (poliol + isocianato) que resultou em compósitos mais resistentes com baixo índice de expansão (Figura 14).

O soquete foi então retirado no molde e, para acabamento, foi lixado e pincelado com três camadas da resina de mamona. Após o tempo de cura do acabamento o protótipo foi montado com seus respectivos componentes e testado (Figura 15).
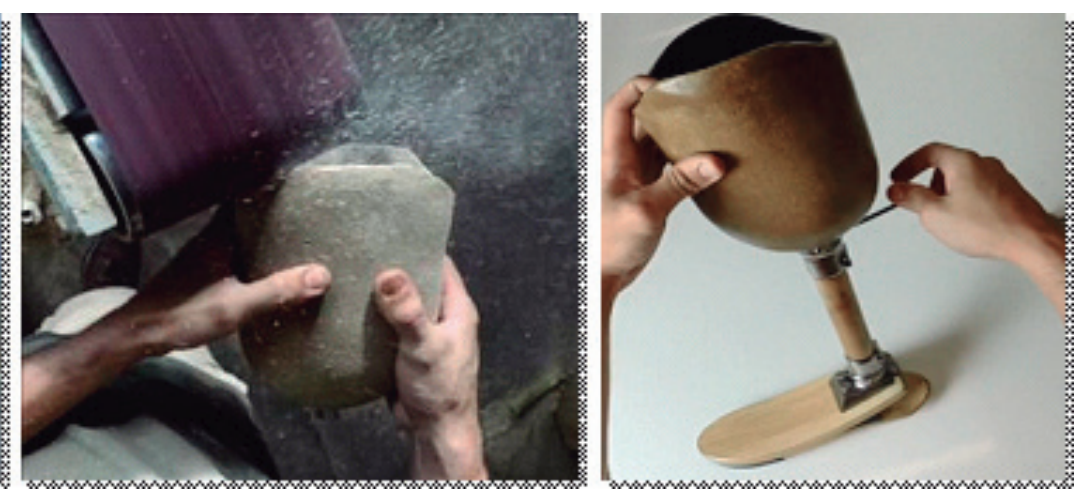


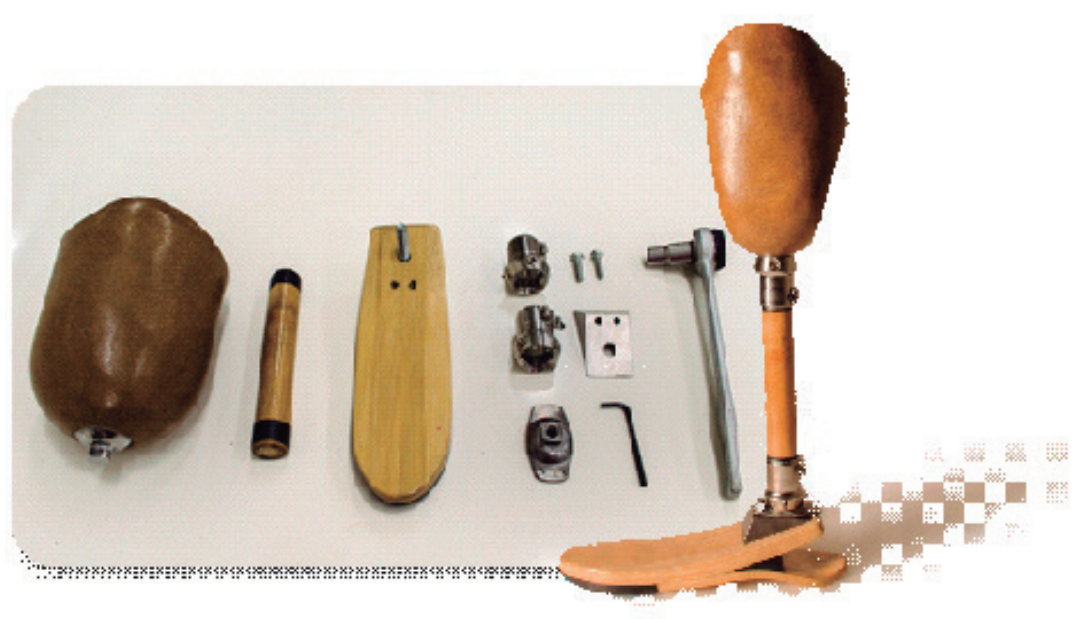

O resultado final do projeto é um protótipo funcional para apresentação da proposta, análises estéticas, físicas e mecânicas (Figura 16). Para testes biomecânicos deverá ser produzido um protótipo projetado para um usuário real.

\section{DISCUSSÃO E CONSIDERAÇÕES FINAIS}

De modo geral é possível concluir que o protótipo resultante atendeu as expectativas nos quesitos: estética, acabamento, compatibilidade do material com outros componentes e trabalhabilidade, comprovando a viabilidade construtiva da prótese e a eficiência do método de fabricação de BLaC proposto por Ramos (2014). É válido ressaltar também que a metodologia criada para fabricação do BRFB também se mostrou apropriada visto que o compósito resultante, que será caracterizado e ensaiado, mostrou-se um biocompósito de fácil laminação, excelente acabamento e relativa resistência a choques e riscos.

A princípio, todos os materiais demonstraram facilidade de trabalho, excelentes acabamento e resistência a riscos, proporcionado pela camada final de resina. Além da proteção e resistência extra, o acabamento com a resina também impermeabilizou os produtos visto que, segundo a fabricante $\mathrm{KEHL}{ }^{\circledR}$ - Indústria e Comércio, testes realizados de acordo com as normas NBR-9779/87; NBR-9779/95 e NBR-10787/94 comprovam a capacidade de impermeabilização das resinas.
Figura 16: Protótipo montado finalizado, peças da prótese desmontada (soquete, cilindro e pé protéticol, componentes modulares e ferramentas necessárias para montagem e ajuste da prótese. 
A princípio pode-se concluir que a utilização de fibras naturais como principais materiais na confecção de próteses e órteses, substituindo os materiais atualmente utilizados, pode minimizar os problemas existentes nos campos de protetização e ortopedia, como alto custo dos produtos, utilização de materiais não sustentáveis, desconforto térmico, entre outros.

Serão realizados estudos futuros baseados na norma ISO 10328 e outras normas específicas para compósitos com o intuito de verificar as propriedades mecânicas do BLaC utilizado no cilindro e pé protético e do compósito BRFB criado para o soquete.

Durante todos os processos, foram coletados dados como tempo de uso de máquina, peso dos materiais, especificações de maquinário, entre outras informações relativas aos insumos necessários para a produção do protótipo. Isto permite realizar uma estimativa de custo, que pode ser fundamental para desenvolvimento futuro das estratégias de negócio.

Com a metodologia e os materiais utilizados neste estudo, outros modelos de próteses ou órteses podem ser confeccionados. Os resultados até agora alcançados permitem confirmar a viabilidade da aplicação de biocompósitos de bambu na produção de próteses transtibiais endoesqueléticas.

Além dos aspectos apresentados, este estudo também teve o propósito de contribuir para o aumento do material científico a respeito do bambu e fomentar a cooperação entre profissionais, como designers industriais, fisioterapeutas e engenheiros, a fim de explorar o potencial do bambu como material resistente, de alto valor agregado e ecologicamente correto.

\section{AGRADECIMENTOS}

Este trabalho foi realizado com financiamento da Coordenação de Aperfeiçoamento de Pessoal de Nível Superior - CAPES e apoio da KEHL ${ }^{\circledR}$ Indústria e Comércio, de São Carlos - SP.

\section{REFERÊNCIAS}


AMERICAN SOCIETY FOR TESTING AND MATERIALS. Standard Terminology for Composite

Materials. American Society for Testing and Materials. West Conshohocken. 2007. (D3878-07)

AMERICAN SOCIETY OF MATERIALS. ASM Handbook - Composites. Materials Park, Ohio:

American Society of Metals, v. 21, 2001

BOCCOLINI, F. Reabilitação: amputados, amputações, próteses. 2. ed. São Paulo: Guanabara, 2000

CARVALHO, J. A. Amputação de membros inferiores: em busca da plena reabilitação. São Paulo: Manole, 2003.

CAVALCANTI, A. L.; PRETO, S. C. S.; FIALHO, F. A. P. \& de FIGUEIREDO, L. F. G. Design para a sustentabilidade: um conceito Interdisciplinar em construção. Projetica, v. 3, n. 1, p. 252-263, 2012

DOUGHERTY, P. J. Transtibial amputees from the Vietnam War. Twenty eight year follow up. J Bone Joint Surg Am. 83-A. 383-389, 2001.

DUDEK, N. L.: MARKS, M. B.: MARSHALL, S. C.: CHARDON, J. P. Dermatologic conditions associated with use of a lower-extremity prosthesis. Archives of physical medicine and rehabilitation, v. 86, n. 4, p. 659-663, 2005.

ELIAS, M. P.; MONTEIRO, L. M. C.; CHAVES C. R. Acessibilidade a benefícios legais disponiveis no Rio de Janeiro para portadores de deficiência física. Laboratório de Disfunção Miccional do Instituto Fernandes Figueira/Fundação Oswaldo Cruz. 2007

FIKSEL, J. Design for environment: creating eco-efficient products and processes. New York: McGraw-Hill, 1996

GAUTHIER-GAGNON C., GRISE MC. Tools for outcome measurement in lower limb amputation. Montreal: University of Montreal, 2001.

GHOSEIRI, K.; SAFARI, M. R. Prevalence of heat and perspiration discomfort inside prostheses: literature review. Journal of rehabilitation research and development, v. 51, n. 6, p. 855, 2014.

HACHISUKA K.; MATSUSHIMA Y.; OHMINE S.; SHITAMA H.; SHINKODA K. Moisture permeability of the total surface bearing prosthetic socket with a silicone liner: is it superior to the patellatendon bearing prosthetic socket? JUoeh 23:225-232. 2001

IIDA, I, BUARQUE, L. M. Ergonomia: projeto e produção. 3. ed. São Paulo: Blucher, 2016.

O'SULLIVAN, S. B; SCHIMITZ, T. J. Fisioterapia: avaliação e tratamento. 2. ed. São Paulo: Manole, 1993

KHAN, G. M. A.; YILMAZ, N. D.; YILMAZ, K. Okra fibers: potential material for green biocomposites. In: Green biocomposites. Springer International Publishing, p. 261-284. 2017.

KLUTE G. K.; ROWE G. I.; MAMISHEV A. V.; LEDOUX W. R. The thermal conductivity of prosthetic sockets and liners. Prosthet Orthot Int 31:292-299. doi: 10.1080/03093640601042554. 2007

KLUTE G. K.; KANTOR C.; DARROUZET C.; WILD H.; WILKINSON S.; IVELJIC S.; CREASEY G. Lower-limb amputee needs assessment using multistakeholder focus-group approach.J Rehabil Res Dev; 46(3):293-304. 2009.

KOC, E.; TUNCA, M.; AKAR, A.; ERBIL, A. H.; DEMIRALP, B. \& ARCA, E. Skin problems in amputees: a descriptive study. International journal of dermatology, v. 47, n. 5, p. 463-466, 2008.

KRAMER A.; SARDO K.; SLOCUMB W. Analysis of bamboo reinforced composites for use in orthotic and prosthetic application. American Academy of Orthotists \& Prosthetists, Washington. (2015).

LAKE, C. R; SUPAN, T. J.The Incidence of dermatological problems in the silicone suspension sleeve user. JPO: Journal of Prosthetics and Orthotics. (C) 1997 American Academy of Orthotists \& Prosthetists. 9(3):97-106. 1997. 
LEVY, S. W. Amputees: skin problems and prostheses. Cutis, v. 55, n. 5, p. 297-301, 1995

LIMA, M. A. M. Introdução aos materiais e processos para designers. Rio de Janeiro: Editora Ciência Moderna, 2006.

LÖBACH, B. Design industrial - Bases para a configuração de produtos industriais. São Paulo: Blucher, 2001.

LÓPEZ, O. H. Bamboo, the gifts of the gods. Columbia, Bogota: D'vinni, 2003.

LYON, C. C.; KULKARNI, J.; ZIMERSONC, E.; VAN ROSS, E.; BECK, M. H. Skin disorders in amputees. Journal of the American Academy of Dermatology, v. 42, n. 3, p. 501-507, 2000

MAHAJAN, G. V; AHER, V. S. Composite material: a review over current development and automotive application. International Journal of Scientific and Research Publications, v. 2, Issue $11,2012$.

MAK, A. F. T.; ZHANG, M.; BOONE, D. State-of-the-art research in lower-limb prosthetic biomechanics-socket interface: a review. Journal of rehabilitation research and development, $v$. 38, n. 2, p. 161,2001

MANOHAR, R. N. Study on use of natural ber composites in prosthetic. Technical report, BITPilani, p. 1-21. 2016.

MEULENBELT, H. E.; GEERTZEN, J. H.; JONKMAN, M. F. \& DIJKSTRA, P. U. Skin problems of the stump in lower limb amputees: 1. A clinical study. Acta dermato-venereologica, v. 91, n. 2, p. 173-177, 2011.

NAYLOR, P. F. D. The skin surface and friction. British Journal of Dermatology, 67: 239-248. 1955.

Peery J. T.; Ledoux W. R.; Klute G. K. Residual limb skin temperature in transtibial sockets. J Rehabil Res Dev 42:147-154. 2006.

PELLICANO, M.; PACHEKOSKI, W.; AGNELLI, J. A. M. Influência da adição de amido de mandioca na biodegradação da blenda polimérica PHBV/Ecoflex ${ }^{\circledR}$. Polímeros, São Carlos, v. 19, n. 3, p. 212-217, 2009.

PELTIER, F.; SAPORTA, H. Design sustentável: caminhos virtuosos. São Paulo: Senac, 2009.

PINTO, M. A. G.; ASTUR, N. F.; GUEDES, J. P. B.; YAMAHOKA, M. S. O. Ponte óssea na amputação transtibial. Revista Brasileira de Ortopedia. v. 33, n. 7, p. 525-31, 1998.

RAMOS, B. P. F. Metodologia de curvatura de bambu laminado colado (BLaC) para fabricação de mobiliário - diretrizes para o design, Dissertação (Mestrado) - Universidade Estadual Paulista. Faculdade de Arquitetura, Artes e Comunicação, Bauru, 2014, 114p.

SANTOS, L. F.; FRITZEN, P. G.; GONÇALVES, B. R.; DE MELO, S. A. \& DA SILVA, V. F. Perfil das amputações de membros inferiores de usuários cadastrados na associação de deficientes físicos de Apucarana. Saúde e Pesquisa, 3(1). 2010

SCHOPPEN, T.; BOONSTRA, A.; GROOTHOFF, J. W.; DE VRIES, J.; GÖEKEN, L. N. \& EISMA, W. H. Physical, mental and social predictors of functional outcome in unilateral lower-limb amputees. Archives of physical medicine and rehabilitation, v. 84, n. 6, p. 803-811, 2003.

TARGA, G. N. compósito reforçado por laminado de bambu com matriz de poliuretano e epóxi: desenvolvimento, produção e caracterização mecânica. 105p. Dissertação de Mestrado, Ciência e Engenharia de Materiais - Universidade Federal de Santa Catarina, Florianópolis, 2011.

TINTLE, S. M.; FORSBERG, J. A.; KEELING, J. J.; SHAWEN, S. B. \& POTTER, B. K. Lower extremity combat-related amputations. Journal of surgical orthopaedic advances, v. 19, n. 1, p. 35, 2010

TOOMS, R. E. General principles of amputation. Campbell W. C.; Campbell's operative orthopaedics. 7. ed. New York, NY: Mosby; 1987.

ZIEGLER-GRAHAM, K.; MACKENZIE, E. J.; EPHRAIM, P. L.; TRAVISON, T. G. \& BROOKMEYER, R. Estimating the prevalence of limb loss in the United States: 2005 to 2050. Archives of physical medicine and rehabilitation, v. 89, n. 3, p. 422-429, 2008. 